\title{
Modulating attentional states by EEG-based neurofeedback
}

Moritz Grosse-Wentrup, Technische Universität München

\begin{abstract}
In recent work, we have presented empirical evidence that attentional networks are associated with a subject's capability to operate a brain-computer interface (BCI) (Grosse-Wentrup \& Schölkopf, Journal of Neural Engineering, 2012; Grosse-Wentrup et al., NeuroImage, 2011).

Here, we present results from a neurofeedback study, in which 19 healthy subjects were trained to induce an attentional state beneficial for operating a BCI. Specifically, subjects received online feedback on the amplitude of parietal gammarange (55-85 Hz) oscillations by means of EEG in conjunction with online beamforming. Subject reports indicate that successful modulation of parietal gamma-power was achieved by shifting between states of focused attention and relaxed wakefulness.

We further found modulation of parietal gamma-power to be negatively correlated with the amplitude of the sensorimotor-rhythm (SMR). As a strong SMR is known to be beneficial for operating a BCI (Blankertz et al., NeuroImage, 2010), our results suggest that volitional attenuation of parietal gamma-power may result in enhanced classification accuracies in SMR-based BCIs. We discuss these results in terms of potential causal relations between parietal- and sensorimotor-areas.
\end{abstract}

